\title{
Correspondencia
}

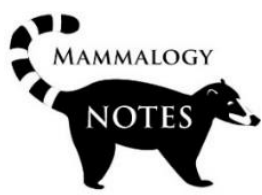

\section{COLECCIÓN DE MAMÍFEROS DEL INSTITUTO HUMBOLDT (IAvH-M)}

\author{
Julián Lozano-Flórez*iD ${ }^{1 *}$ Sebastián Cifuentes-Acevedo' ${ }^{10}$, Kevin Borja-Acosta'(i) \\ Carolina Gómez-Posada'ii
}

1 Instituto de Investigación de Recursos Biológicos Alexander von Humboldt. Carrera 8 \# 15 - 08, Villa de Leyva, Boyacá

*Correspondencia: lozanoflorezbio@gmail.com, alozano@humboldt.org.co

\section{Resumen}

La colección de mamíferos del Instituto de Investigación de Recursos Biológicos Alexander von Humboldt es la tercera colección de mayor número de especímenes en el país con 9.471 determinados en 400 especies, 212 géneros, 51 familias y 14 órdenes, procedentes de ocho países, siendo Colombia el de mayor representatividad $(66,5 \%$ de la diversidad registrada para el país en este grupo). Los órdenes mejor representados son Chiroptera, Rodentia y Primates. Alberga un neotipo de Cebus albifrons albifrons, un paratipo de Aotus hershkovitzi y un paratipo de Vampyressa sinchi. La colección ha sido un referente para el estudio de mamíferos colombianos y se continúan realizando esfuerzos para mejorar el estado curatorial y la información asociada a los especímenes.

Palabras clave: Colección biológica, especímenes, clase Mammalia, mamíferos en Colombia, Sur América.

\section{Abstract}

The Mammal Collection of the Humboldt Institute is the third largest collection in the country and houses 9.471 specimens. These belong to 400 species, 212 genera, 51 families and 14 orders, from eight countries, Colombia the one with a higher representation $(66,5 \%$ of the species registered in the country). The best represented orders are Chiroptera, Rodentia, and Primates. The collection houses one neotype of Cebus albifrons albifrons, a paratype of Aotus hershkovitzi and a paratype of Vampyressa sinchi. The collection has been a benchmark for the study of the Colombian mammals and the Institute continues its effort to improve the curatorial status and the information associated to the specimens.

Key words: Biological Collection, specimens, Class Mammalia, Colombian mammals, South America.

La colección de mamíferos del Instituto de Investigación de Recursos Biológicos Alexander von Humboldt (IAvH-M) fue heredada de la unidad Científica Federico Medem del extinto Instituto de Recursos Naturales (INDERENA). En el año de 1995, el Instituto Humboldt recibió la colección (Arbeláez-Cortés et al. 2017) y en consecuencia se realizó un cambio en el 
acrónimo de los especímenes catalogados, los cuales pasaron de ser IND-M a IAvH-M. Además, fue trasladada desde la ciudad de Bogotá a Villa de Leyva, Boyacá, donde hoy se encuentra. En la actualidad, la colección alberga 9.471 especimenes de mamíferos pertenecientes a 14 órdenes, 51 familias, 212 géneros y 400 especies (Tabla 1). Los órdenes mejor representados son Chiroptera y Rodentia con 154 y 107 especies respectivamente (Tabla 1). También se resalta la representatividad del orden Primates con 37 especies de las 40 reportadas para Colombia. Los especímenes de la colección provienen de ocho países: Brasil (14), Canadá (3), Ecuador (5), Perú (43), Estados Unidos (112), México (3), Venezuela (4), y Colombia (9.285). En Colombia, los especímenes provienen de 2.072 localidades de 32 departamentos.

Un total de 75 especímenes están identificados a nivel de clase, 72 a orden, 68 a familia, 7 a subfamilia, 1.136 a género, 7.749 a especie y 364 a subespecie. Para Colombia están representadas 351 especies que corresponden al 66,5\% de las registradas para el país (Ramírez-Chaves et al. 2019), sin embargo, se considera que el número de especies puede ser mayor, debido a una alta cantidad de especímenes que necesitan ser revisados en su identificación taxonómica.

TABLA 1. Órdenes, familias, géneros y especies de mamíferos depositados en la colección de mamíferos del Instituto Humboldt (IAvH-M). No se incluyen 75 especimenes identificados a nivel de Clase: Mammalia. *Número de especies de mamíferos registradas en Colombia según la lista de Ramírez-Chaves et al. (2019).

\begin{tabular}{cccccc}
\hline Orden & Familias & Géneros & Especies & $\begin{array}{c}\text { *Especies } \\
\text { en Colombia }\end{array}$ & $\begin{array}{c}\text { Número de } \\
\text { Especimenes }\end{array}$ \\
\hline Didelphimorphia & 1 & 10 & 21 & 18 & 210 \\
\hline Paucituberculata & 1 & 1 & 1 & 1 & 26 \\
\hline Cingulata & 1 & 3 & 6 & 6 & 34 \\
Pilosa & 3 & 5 & 7 & 7 & 82 \\
\hline Sirenia & 1 & 1 & 2 & 2 & 6 \\
\hline Soricomorpha & 1 & 2 & 7 & 4 & 76 \\
Chiroptera & 9 & 72 & 154 & 146 & 5.301 \\
Carnivora & 7 & 24 & 31 & 23 & 488 \\
Perissodactyla & 1 & 1 & 3 & 3 & 51 \\
\hline Artiodactyla & 3 & 9 & 16 & 12 & 347 \\
Cetacea & 4 & 7 & 7 & 6 & 41 \\
\hline Primates & 5 & 15 & 38 & 37 & 785 \\
Rodentia & 13 & 60 & 107 & 83 & 1.900 \\
Lagomorpha & 1 & 2 & 5 & 3 & 49 \\
Total general & 51 & 212 & 400 & 351 & 9.396 \\
\hline
\end{tabular}

Los datos biológicos y geográficos asociados a los especímenes de la colección de mamíferos IAvH-M se encuentran publicados y disponibles en los siguientes enlaces: Instituto Humboldt (http://i2d.humboldt.org.co/ceiba/resource.do?r=mamiferos_iavh) y SiB Colombia (http:/ /ipt.biodiversidad.co/iavh/resource.do?r=mamiferos_iavh).

Actualmente, la colección alberga el neotipo del mono cariblanco Cebus albifrons albifrons Humboldt, 1812 (IAvH-M-2844) (Defler \& Hernández-Camacho 2002). También cuenta con un paratipo del mono nocturno Aotus hershkovitzi Ramírez-Cerquera 1983 (IAvH-M-4140), 

que es considerado actualmente un sinónimo de Aotus lemurinus lemurinus Defler \& Bueno 2001. Finalmente, la colección contiene un paratipo del murciélago quechua de orejas amarillas Vampyressa sinchi Tavares, Gardner, Ramírez-Chaves, \& Velazco. 2014 (IAvHM-2282) (Figura 1).
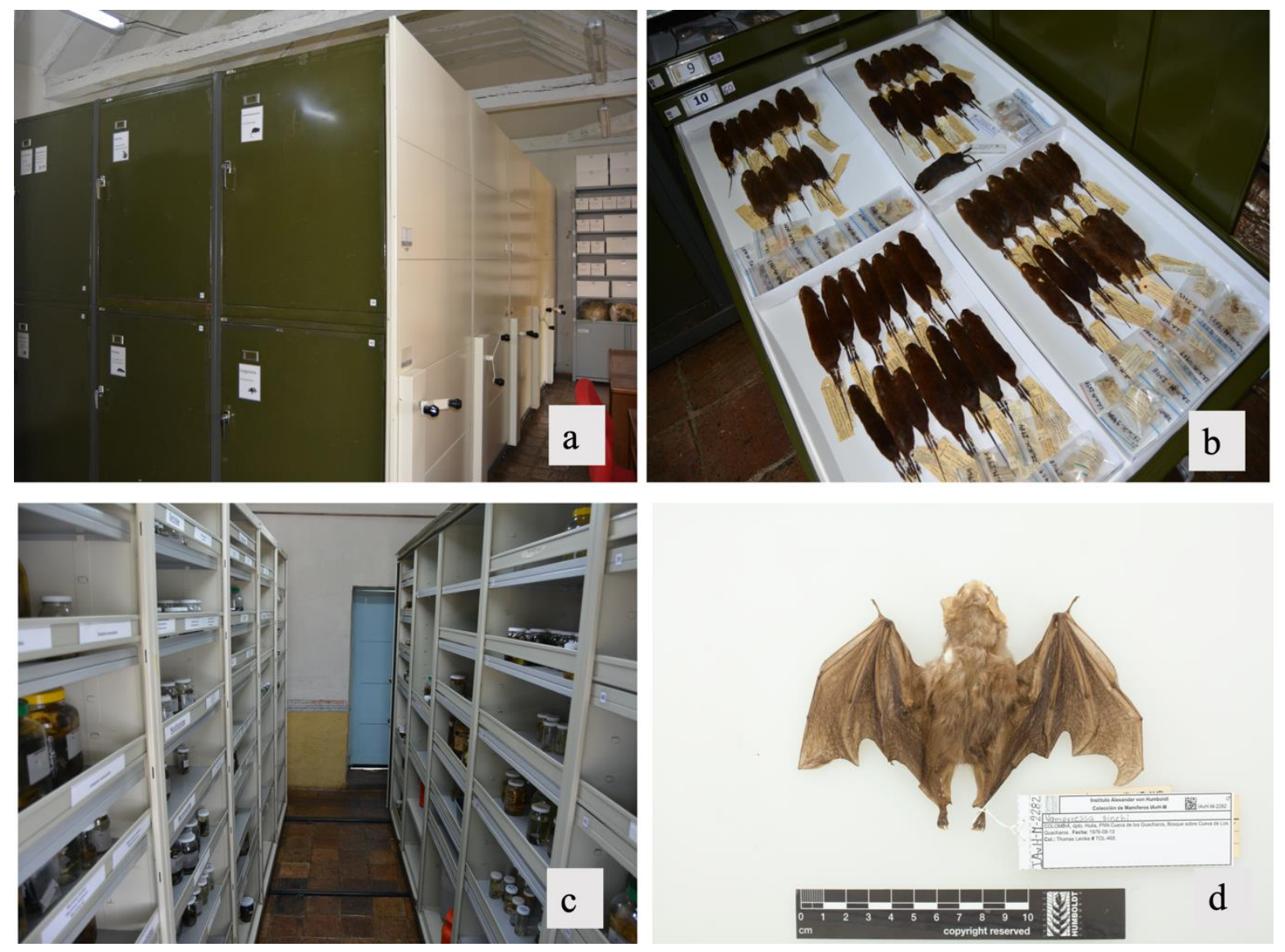

FIGURA 1. Detalle de la organización en la colección de mamíferos IAvH-M. a) Compactadores de especímenes preservados en seco, b) Organización de especímenes de mamíferos pequeños, c) Compactador y entrepaños de los especímenes preservados en líquido, d) Paratipo de Vampyressa sinchi (IAvH-M-2282).

Entre los principales recolectores se encuentran Jorge Hernández Camacho "el Mono Hernández", Hernando Chiriví, Jorge Morales, José Vicente Rodríguez, Luz Myriam Moreno, Thomas Lemke, Wendy Towsend, Yaneth Muñoz, Fabio Quevedo, Fernando Forero, además de múltiples personas más que han contribuido a la colección. Los especímenes recolectados desde etapas tempranas y que hoy alberga la colección, han contribuido a través de los años a documentar la riqueza de mamíferos colombianos (Alberico et al. 2000; Solari et al. 2013), así como para brindar información de línea base para la creación de los Parques Nacionales Naturales en el país (Córdoba-Córdoba 2009).

Durante años la colección de mamíferos del Instituto Humboldt ha sido un referente para el estudio de los mamíferos en Colombia. Esto se ha visto reflejado en el uso de cerca de 700 especímenes en más de 60 publicaciones científicas entre artículos y libros, realizadas por investigadores nacionales e internacionales. Adicionalmente, en el último año se han construido redes de colaboración y se han incrementado las actividades de curaduría, 
revisión completa, manejo y divulgación que permitirán garantizar la calidad de la información asociada a los especímenes y un mejor uso de estos por la comunidad académica.

\section{AGRADECIMIENTOS}

A Socorro Sierra, Janeth Robles y Guillermo Cárdenas quienes contribuyeron enormemente en las actividades recientes de mejoramiento de la colección de mamíferos. También a todas las personas que a través del tiempo contribuyeron al mantenimiento de la colección de mamíferos del Instituto Humboldt

\section{REFERENCIAS}

Alberico M, Cadena A, Hernández-Camacho J, Muñoz-Saba Y. 2000. Biota Colombiana. 1, 43 - 75

Arbeláez-Cortés E, Acosta-Galvis AR, DoNascimiento C, Espitia-Reina D, González-Alvarado A, Medina CA. 2017. Knowledge linked to museum specimen vouchers: measuring scientific production from a major biological collection in Colombia. Scientometrics. 112,1323-1341. https:// doi.org/10.1007/s11192-017-2461-4

Córdoba-Córdoba S. 2009. Historia de la ornitología colombiana: sus colecciones científicas, investigadores y asociaciones. Boletín SAO 19, 1-26

Defler TR, Bueno ML, Hernández-Camacho JI. 2001. Taxonomic status of Aotus hershkovitz: its relationship to Aotus lemurinus lemurinus. Neotropical Primates. 9, 37-62

Defler TR, Hernández-Camacho JI. 2002. The true identity and characteristics of Simia albifrons Humboldt, 1812: Description of neotype. Neotropical Primates. 10, 49-70

Ramírez-Cerquera J. 1983. Reporte de una nueva especie de primate del género Aotus de Colombia. Resúmenes de las Comunicaciones Científicas del IX Congreso Latinoamericano de Zoología, Arequipa, Perú.

Ramírez-Chaves HE, Suárez-Castro AF, Sociedad Colombiana de Mastozoología, Zurc D, Concha Osbahr DC, Trujillo A, Noguera Urbano EA, Pantoja Peña GE, Rodríguez Posada ME, González Maya JF, Pérez Torres J, Mantilla Meluk H, López Castañeda C, Velásquez Valencia A, Zárrate Charry D. 2019. Mamíferos de Colombia. Versión 1.6. Sociedad Colombiana de Mastozoología. Checklist dataset https:// doi.org/10.15472/kl1whs Descargado Marzo 2020.

Solari S, Muñoz-Saba Y, Rodríguez-Mahecha JV, Defler TR, Ramírez-Chaves HE, Trujillo F. 2013. Riqueza, Endemismo y Conservación de los Mamíferos de Colombia. Mastozoología Neotropical. 20, 301-365

Tavares VDC, Gardner AL, Ramírez-Chaves HE, Velazco PM. 2014. Systematics of Vampyressa melissa Thomas, 1926 (Chiroptera: Phyllostomidae), with descriptions of two new species of Vampyressa. American Museum Novitates. 3813, 1-27.

Editor: Miguel E. Rodríguez-Posada

Recibido 2020-05-08

Revisado 2020-05-14

Aceptado 2020-06-09

Publicado 2020-07-12 